\title{
THE ARCHITECTURE OF INFORMATION FUSION SYSTEM IN GREENHOUSE WIRELESS SENSOR NETWORK BASED ON MULTI-AGENT
}

\author{
Wenting Zhu ${ }^{1}$, Ming Chen ${ }^{1, *}$ \\ ${ }^{1}$ Department of Information Technology, Shanghai Ocean University, Shanghai, P. R. China \\ 200090 \\ * Corresponding author, Address: Department of Information Technology, Shanghai Ocean \\ University, Shanghai 200090, P. R. China, Tel: +86-13371935523, Fax: +86-21- \\ 65710862,Email:mchen@shou.edu.cn
}

\begin{abstract}
In view of current unprogressive situation of factory breeding in aquaculture, this article designed a standardized, informationized and intelligentized aquaculture system, proposed a information fusion architecture based on multi-agent in greenhouse wireless sensor network (GWSN), and researched mainly the structural characteristic of the four-classed information fusion based on distributed multi-agent and the method to construct the structure inside of every agent.
\end{abstract}

Keywords: GWSN, multi-agent, information fusion

\section{INTRODUCTION}

For a long time aquaculture in china mostly uses traditional ways that consume lots of resources, especially shrimp breeding uses the mode of production of high-density, high devotion, high yield and a high rate of water change, at the same time the lower level of management mainly depends on the experiences. It is Inconsistent with principles and policies in china that vigorously develop digital fishery and accurate fishery. With accelerating of information course in the whole society, networking and digital information environment forms in accelerating. To achieve

Please use the following format when citing this chapter:

Zhu, W. and Chen, M., 2009, in IFIP International Federation for Information Processing, Volume 295, Computer and Computing Technologies in Agriculture II, Volume 3, eds. D. Li, Z. Chunjiang, (Boston: Springer), pp. 2057-2066. 
comprehensive information system of fisheries and sustainable development has become a great challenge of $21^{\text {st }}$ century. Therefore, standardized production and operation, industrialized and intelligentized greenhouse breeding methods will become one of the directions of the future development in the aquaculture industry.

Therefore, breeding environment has great significance on High-density factory aquaculture. Timely monitoring and controlling the changes in water quality factors and improving the condition of breeding water quality can effectively enhance the output and efficiency of breeding. based on the establishment of a multi-sensor nodes wireless sensor network (Akyildiz et al., 2002) of real-time monitoring system, this paper researches the technology of data fusion (Cheng Hongbing et al., 2006) on deferent sampling frequency of sensor layer under different types of Integrated sensor nodes such as temperature, $\mathrm{pH}, \mathrm{DO}$, ORP, light, etc, Combines testing data of laboratory, real-time data that collected by the sensor network, sensor data, historical data and growth of biological data collected through offline, data of feeding conditions and Expert knowledge, fuses data from data center layer, establishes a waters micro-ecology environment and biological growth model. At the same time, the multi-agent technology will be introduced in all levels of data fusion (Fan Bo et al., 2003). Using the characteristics of autonomy, coordination, self-learning and reasoning of multi-agent technology (Michael et al., 1995), the system can be reasoning, integration and learning by capture data information and state from environment, and it makes a great improve on the Intelligence of data fusion technology. This paper constructs a decision-making model based on multi-agent by information exchange at all levels of data fusion to intelligently control and breed micro-ecological environment and growth process of the whole factory breeding. The model will optimize the breeding technology, reduce production expanse and laboring intensity, increase productivity, achieve the modernization and intelligence of aquaculture, and accelerate the commercialization of aquaculture.

This paper is organized as follows: the 2nd section introduces related works; the 3rd section proposes the architecture of multi-agent information fusion of GWSN; the 4th section brings forward the function and internal structures of each agent; the last section will give a conclusion to the whole system. 


\section{THE ARCHITECTURE OF MULTI-AGENT INFORMATION FUSION SYSTEM IN GWSN}

\subsection{GWSN}

The purpose which we established GWSN is to regulate the water areas and environment of greenhouse, reduce the production-costs and labor intensity, improve the efficiency of production through collect the key factors of relative water areas and greenhouse's environment in real-time, and combine the regulation models of establishing water areas and environment such as chemical analysis laboratory factors, biological growth factors and so on.

An intensive breeding factory is composed by several greenhouses (workshops), every greenhouse workshop is composed by many standardized ponds, and the factory regulates the water quality and the greenhouse environment through the uniform control system. It used a cluster structure when constructed the monitoring network (Wang Xue et al., 2007). It designed a cluster which is managed by a clustering node in a greenhouse workshop; a greenhouse workshop equipped with a number of wireless multi-sensor nodes which connects five sensors: PH, DO, light, dissolved oxygen, temperature sensor and a wireless control node which controls the equipment such as water pump, aerobics machine, lighting, curtain and so on. Several clusters are connected with central monitoring room. The features of GWSN which are different from other wireless sensor networks because:

(1)Cluster Structure. The wireless sensor nodes only transmits data to cluster node and send control order to the control node in the same cluster, and the sensor nodes do not transmit the messages to each other; there is only a control node in a cluster; the control node can receive the order of sensor nodes in this cluster, the control order of cluster nodes and the control order of central node passed through cluster node.

(2)It has good power supply, and the node which is easy to maintain have the small possibility to failure; but it greatly influences the stability of the sensors' signal and the control equipment.

(3)A wireless sensor node integrated a number of sensors; a control node integrated a number of control equipment.

\subsection{Construction of multi-level information fusion system}

According to the features of the wireless sensor network system in factory 
breeding, this article researches multi-source information fusion technology (Hu Xuejun et al., 2002) such as a variety of sensors information, chemical analysis information, production information and the metabolism of biological growth under the high-density breeding and closed environment, and establishes multi-level information fusion system, shown as Fig.1.

(1)First-class fusion is information fusion of sensor and information processing. Every sensor collects information on single parameter, and mainly solves how to keep the collected information according with the actual situation. The reasons which mainly influence the data different from the fact are: (i) Environmental noise influence greatly on sensor's signal. (ii) The long-term use of sensor will lead to the surface passivation, reduce the signal sensitivity and possibly appear the drift phenomenon. In the first-class fusion, it mainly deals with the data-different situation.

(2)Second-class fusion is information fusion of several sensor nodes. The wireless multi-sensors node include the water quality sensor such as $\mathrm{PH}$, salinity, DO, ORP and greenhouse environment sensor such as lighting, temperature and so on. Through long-term experiments and practical breeding productions, it founds that different kinds of water quality parameters have the different relativity under different situation. We focus on analyzing the mutual-change relation models of every sensor's parameter when the parameter of water quality is under the external condition such as feed delivery, medication, control water quality and change temperature, illumination, then establishing the rules of changing water quality's parameter and judging whether the collected data every time is same as the current actual water quality and greenhouse environment in real-time according to the rule-based learning. Once the data relativity disaccords, the system should establish the alarm model of sensor nodes and send the order to the control nodes to regulate the water environment.

(3)Third-class fusion is information fusion of greenhouse workshop (cluster node). The focus of third-class fusion is that: (i) It preprocesses the information of the several wireless sensor nodes, then sends the preprocess results to the central control node in order to accomplish the variance analysis and the weighted average analysis; (ii) It combines relation models of sensors' parameter, researches the relation between the change of ammoniac nitrogen, nitroso nitrogen, COD in the water and PH, salinity, Do; (iii) according to the environmental conditions of the whole greenhouse, it establishes the control model under the exceptional condition and send the control order to the control nodes.

(4)Fourth-class fusion is information fusion of data center (central node). In the information fusion of this class, it should deal with three problems: (i) Filter the mainly attribute. First we should find the mainly relative attributes from a mass of attributes in order to find out the mainly influencing attribute in every kind of parameters; then we consider to design a statistical learning way via setting up confidence interval in order to regulate the weight of 
attributes step by step and achieve the propose of filtering mainly attributes; (ii) Multi-model analysis. Every attribute has complex relationships, some have the statistics regularity such as water quality's parameter and the delivery number of different feeds, some can bring the specific rules such as biological metabolism rule, some regularity hides in a mass of information such as the influence of $\mathrm{PH}$ and ammoniac nitrogen in production of microalgae and the component of breeding biologic metabolites. We use the method of data mining such as fuzzy neural network model, multivariate statistics and rule-based learning according to the different conditions to establish the different relation model. (iii) Decision analysis. We make every kind of regulated parameters of greenhouse factory breeding as the target of decision, and establish the decision analysis model of multi-target according to every attribute relation models to make decision on every kind of target parameter, then regulate the water quality via regulation of relative parameter to establish the environment of greenhouse and water quality which is suitable for biological growth.

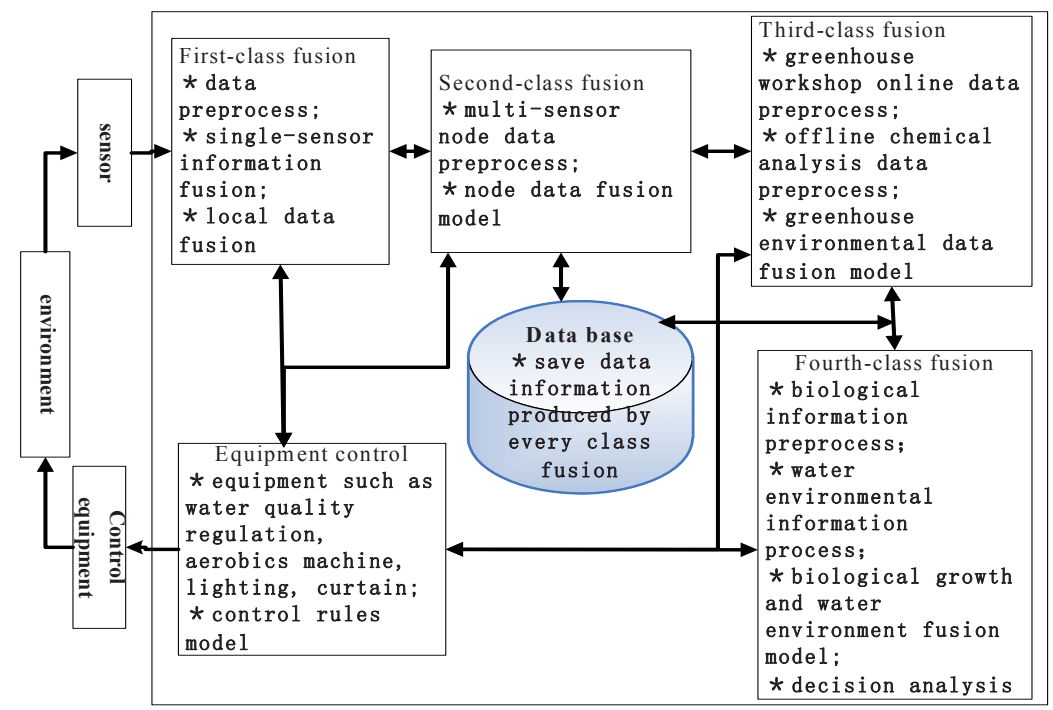

Fig.1: structure of multi-level information fusion in greenhouse monitoring network

\subsection{Design of multi-agent information fusion system in GWSN}

According to the features of the information fusion system in GWSN, it designs a multi-agent system (Wang Jun et al., 2008) to reflect the autonomy, adaptability and coordination of information fusion in different level, specific structure shown as Fig. 2. 
In the whole multi-agent system, it emphasizes the independent work of every local agent to solve the own problem and the functional requirements; the adaptability of agent is an ability of adapting local requirement and selfregulation. At the same time, according to the requirement of every class information fusion, all agents communicate and coordinate each other under the coordination of routing coordination agent. The mainly function and mutual relation of every agent in GWSN as follows:

- Single Sensor Information Process Agent: solve the information drift problem of sensors; collect real-time information then send the information and equipment failure information to local multi-sensor information fusion agent.

- Local Multi-Sensor Information Fusion Agent: establish the relation of multi-sensor's real-time parameter to form the environment of biological growth and the required area of water quality in local pond area, then send the fusional environmental information to area information fusion agent and send the equipment control order to equipment control agent.

- Area Information Fusion Agent: combine the real-time information of different multi-sensor information fusion and water environment regulation model which is formed by ammoniac nitrogen, nitroso nitrogen, COD analyzed by lab termly in the range of local greenhouse, then send the fusional greenhouse environmental information to central decision agent and send the control order to control agent according to the requirement.

- Central Decision Agent: combine the environmental information of area information fusion agent, biological growth information and production output information, establish the environmental optimization model to form the decision analysis system of production output, biological growth and environment control, and send the control order to equipment control agent according to the requirement of environmental optimization.

- Routing Coordination Agent: according to the communication protocol and the features of GWSN, it can create the routing rules between different agents and send decision according to the requirement of different agent packet.

- Equipment Control Agent: generally speaking, the control order of multi-sensor nodes is instant control orders that the one or several parameters of water quality or environment is in the critical range (alarm range) of biological growth. The control order of area information fusion agent is based on the every kind of real-time information of greenhouse water environment and lab information. The control order of central decision agent is based on the optimal environmental requirement of biological growth and requirement of production decision, so the control order of every class has the different priority. Equipment control agent should create the rule priority of different equipment control and form the rule-base, when the equipment does not work, equipment control agent will send the alarm of fail information to the relative node. 


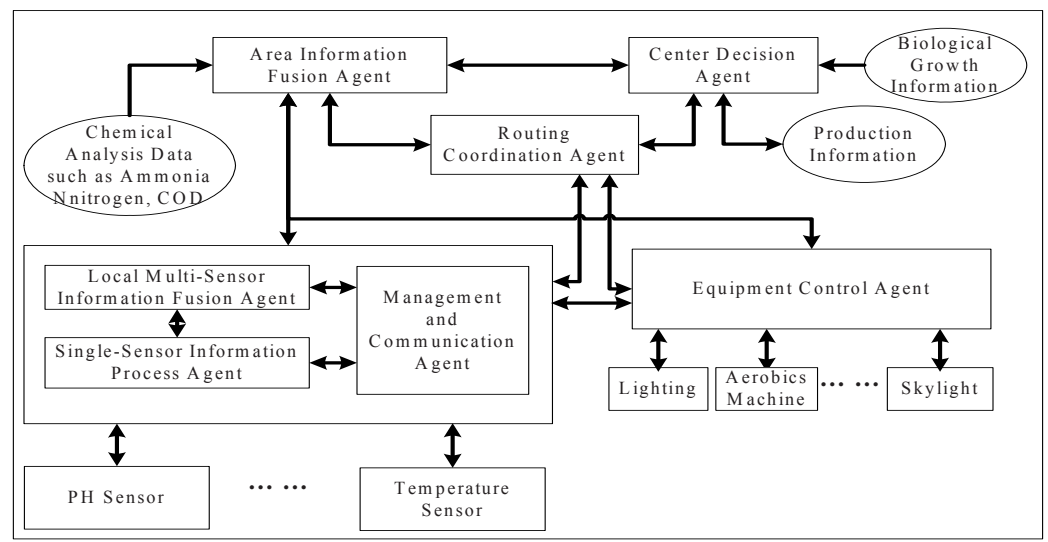

Fig.2: the architecture of multi-agent information fusion of GWSN

\section{RESEARCH ON THE STRUCTURE OF AGENTS IN MULTI-AGENT SYSTEM}

\subsection{The internal structure of agent}

Based on the features of greenhouse multi-agent information fusion system, the independent ability and coordination relation of every kind of agent (Kraus, 1997), it considers that the inner structures of agent includes data cache, triggered events which trigger agent, knowledge base $(\mathrm{KB})$ or rule base $(\mathrm{RB})$ of agent which process the information fusion intelligently, processing module which process the inside of agent according to knowledge rules and triggered events, coordination module which coordinate the inner components and data exchange according to relative strategies, outer communication interface and so on. The structure is shown as Fig.3.

And with all the modules, different Agents have different inside functions, their internal structures of every module are as follows:

- $\quad<$ Agent $>$ : : $=<$ data cache $><$ event trigger $><\mathrm{RB}, \mathrm{KB}><$ processing module $><$ coordination processing $><$ outer communication interface $>$

- $\quad<$ data cache $>$ : : = [local data][outer data]

- $\quad<$ event trigger $>$ : : =<real-time information trigger of sensors $><$ failure information trigger $><$ trigger control order $>$

- $\quad<\mathrm{RB}, \mathrm{KB}>\mathrm{:}:=$ [data structure][routing rules] [fusion arithmetic][control trigger rules]

- $\quad<$ processing module $>$ : : $=<$ information collection $><$ information 
transmits $><$ equipment control signal $><$ routing and packet processing $>$

- $<$ coordination module $>:$ : $=<$ coordination protocol $><$ coordination strategy $><$ coordination process $>$

- $\quad$ outer communication interface $>$ : $=<$ information collection communication $><$ failure alarm communication $><$ communication with other agents>

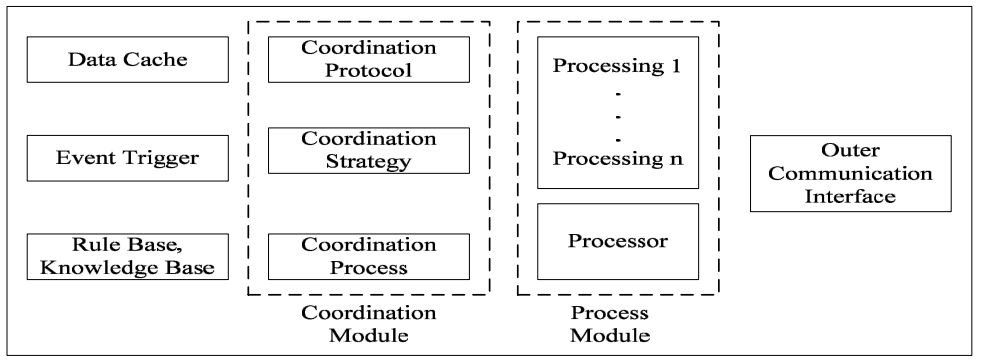

Fig.3: structure mode of agent

\subsection{Establishing RB of Agent}

It is important for each agent to build a RB in information fusion, because different agent corresponds to different level of information fusion and has different function; however, the fusion algorithm is different too. In this section we mainly research on establishing RB of Local Multi-sensor Information Fusion Agent which corresponds to the second-class information fusion. We used mainly rule based reasoning to fuse factors of water quality in Local Multisensor Information Fusion Agent. Every factor such as salinity, PH value, and water temperature was transformed into rules according to the standard range which is suitable for biological growth and saved as two-dimensional form. The basic form of rules is:

\section{IF P THEN Q}

Thereinto, $\mathrm{P}$ represent the condition, $\mathrm{Q}$ represent the result. It means if $\mathrm{P}$ is reached, then does the action $\mathrm{Q}$ defined.

When Local Multi-sensor Information Fusion Agent received data, the system calls the rule of knowledge base and does the fusion processing to data. The pseudo codes of part knowledge are as follows:

If Type of Prawn = JUVENILE

If Water Temperature $>23$ or Water Temperature $<20$ Then Call Alarm Module and Call Control Module

Else Submit Fused Information to Area Information Fusion Agent

Else If Type of Prawn $=$ ADULT

If Water Temperature $>30$ or Water Temperature $<25$ Then Call Alarm Module and Call Control Module

Else Submit Fused Information to Area Information Fusion Agent

If Type of Prawn = JUVENILE 
If Salinity $>6$ or Salinity $<3$ Then Call Alarm Module and Call Control Module Else Submit Fused Information to Area Information Fusion Agent

Else If Type of Prawn $=$ ADULT

If Salinity $>3$ Then Call Alarm Module and Call Control Module

Else Submit Fused Information to Area Information Fusion Agent

Due to the lots of factors affecting water quality, we just listed the rules of water temperature and salinity. After establishment of rule-base, we used RETE algorithm to do the rule based reasoning. It's a high-efficient pattern-matching algorithm. When the Local Multi-sensor Information Fusion Agent receives water quality data, the processor module will call the $\mathrm{RB}$ and do some rule based reasoning to the data. It is worth noting that the processor handles incidents at two levels. Equipment failure is the first process level. When equipment failure, the processor will trigger alarm module for a failure signal. Data fusion is the second process level. The processor calls RETE algorithm to fuse several kinds of water quality factors. If the factor of any kinds is beyond the standard range, the processor will trigger alarm module immediately and send the regulating signal to Equipment Control Agent to regulate the relevant factors of water quality to standard range; if all factors of water quality are in the standard range, the processor will send these water quality data to the superior information fusion. Now I will take out 4 sets of data for analysis from a local multi-sensor node (cluster node) of an adult prawn breeding pond to prove that the second-class information fusion algorithm is reliable and efficient. The data is shown in Table1.

Table 1. Analysis of water quality factors fusion

\begin{tabular}{lllll}
\hline & Temperature & PH & DO & Result \\
\hline 1 & $31.2^{\circ} \mathrm{C}$ & 7.84 & $4.56(\mathrm{mg} / \mathrm{l})$ & Alarm \\
2 & $29.2^{\circ} \mathrm{C}$ & 8.07 & $6.95(\mathrm{mg} / \mathrm{l})$ & Submit \\
3 & $28.2^{\circ} \mathrm{C}$ & 6.94 & $2.57(\mathrm{mg} / \mathrm{l})$ & Alarm \\
4 & $26.6^{\circ} \mathrm{C}$ & 7.65 & $4.48(\mathrm{mg} / \mathrm{l})$ & Alarm \\
\hline
\end{tabular}

We selected the three factors of temperature, $\mathrm{PH}$ and $\mathrm{DO}$ for fusion, and the numerical value beyond the standard range was marked by yellow in table 1 . The first set of data in Table 1 shows that the temperature exceeds the standard range, because the temperature range of adult prawn is $25^{\circ} \mathrm{C}-30^{\circ} \mathrm{C}$. In the second-class information fusion, when the Local Multi-sensor Information Fusion Agent receives the first set of data, the processor will trigger the alarm module after fusion for an alarm signal and conduct the Equipment Control Agent to open the cooling device. So the column of "Result" in table 1 shows "Alarm". The second set of data is in the standard range after fusion, and the processor will send these data directly to the superior information fusion, so the column of "Result" in table 1 shows "Submit". Facts have proved that this approach of information fusion in Local Multi-sensor Information Fusion Agent can process data effectively and regulate the aquaculture environment of prawn to an optimal condition. 


\section{CONCLUSION}

This article researches the modern, intelligentized, factory aquaculture system deeply, and proposes a multi-agent information fusion system structure in GWSN. It focuses on every class information fusion technology and the information fusion structure model based on multi-agent. Due to the characteristics of agent such as autonomy, distribution and coordination, the function of the multi-sensor information fusion system become more powerful and the overall capability of the system is farther improved.

\section{ACKNOWLEDGEMENTS}

This research is supported by National 863 Plan, No. 2006AA10Z253, 2006AA10Z311, 2007AA10Z238 (P. R. China).

\section{REFERENCES}

Akyildiz L F, Su WeiLian, Sankarasubramaniam Y, Cayirci E. A Survey on Sensor Networks. IEEE Communications Magazine, 2002, 40(8):102-114

Cheng Hongbing, Huang Xiao, Yang Geng. Research of Data Fusion System Architecture in Wireless Sensor Network. Computer Science, 2006, 33(7):60-62(in Chinese)

Fan Bo Etc. The Technology of Multi-Agent Introduced to Information Fusion System. Computer Engineering and Applications, 2003(22):100-102(in Chinese)

$\mathrm{Hu}$ Xuejun, Luo Zhongliang. Method of multi-sensor information fusion based on statistics theory. Journal of Transducer Technology, 2002(08):38-43(in Chinese)

Kraus S. Negotiation and cooperation in multi-agent environments. Artificial Intelligence, 1997, 94(1):79-97

Michael W, Nicholas R J. Intelligent agents: theory and practice [J]. Knowledge Engineering Review, 1995, 10 (2):115-152

Wang Jun, Wang Yuanyun. Multi-agent and the Application in the Information Fusion. Computer and Digital Engineering, 2008, 36(3):128-130(in Chinese)

Wang Xue, Wang Sheng, Jiang Aiguo. Cluster-based data fusion decision method in wireless sensor. Control and Decision, 2007, 22(11):1208-1212(in Chinese) 